\title{
Methods and Principles of Limb Salvage Treatment for Malignant Bone Tumors of Extremities
}

\author{
Rui Jiang ${ }^{1}$, Ziyan Zhang ${ }^{2}$, Han $\mathrm{Wu}^{1}$ and Chao Zhang ${ }^{3, \text { a, * }}$ \\ ${ }^{1}$ Department of orthopedics, China-Japan Union Hospital of Jilin University, Jilin 130033, China \\ ${ }^{2}$ Department of orthopedics, The Second Hospital of Jilin University, Jilin 130041, China \\ ${ }^{3}$ Department of ophthalmology, The Second Hospital of Jilin University, Jilin 130041, China \\ azhangchao0701@163.com
}

\begin{abstract}
Keywords: Malignant bone tumors, Limb Salvage Treatment, Neo- adjuvant chemotherapy, Wide resection.
\end{abstract}

\begin{abstract}
The treatment of malignant bone tumors has been maturely developed, while neo-adjuvant chemotherapy and wide resection have been widely promoted. Limb salvage technique has become a classical method to treat the malignant bone tumors of extremities. Domestic treatment of bone tumors has made much progress during the past 20 years. In the paper, the concept of neo-adjuvant chemotherapy, wide resection principles and methods are mainly discussed, and the possible problems are expounded.
\end{abstract}

\section{Introduction}

The treatment of malignant bone tumors has been maturely developed, while neo-adjuvant chemotherapy and wide resection have been widely promoted. Limb salvage technique has become a classical method to treat the malignant bone tumors of extremities. Neo-adjuvant chemotherapy can eliminate the micro metastasis and shrink the primary tumors in the early stage, which benefits the subsequent limb salvage treatment, and provides the information on in vivo chemotherapy sensitivity test by evaluating the reaction of tumors to chemotherapy. The treatment of malignant bone tumors is provided to not only improve the survival rate of patients, but also maintain the good limb function. Limb salvage surgery should first prevent local recurrence, and then maintain as many functions as possible. In the past 20 years, China has made great progresses in the treatment of bone tumors, and extensively promoted the concept of neo-adjuvant chemotherapy and the principles and methods of wide resection, which improves the survival rate of patients with malignant bone tumors significantly. As chemotherapy can reduce the surgical margin of tumor, wide excitation can be implemented on this basis to realize optimal margin resection, which not only keeps the extremities that still function, but also achieves the radical cure for local tumor. The key to this treatment is the selection of suitable surgical margin.

\section{Chemotherapy}

In the past 20 years, the treatment of malignant bone tumors has been greatly improved, which must be significantly attributed to the introduction and gradual bettering of chemotherapy, especially the application of neo-adjuvant chemotherapy. Before chemotherapy was introduced to treat the malignant bone tumors represented by osteosarcoma and Ewing's sarcoma, the common treatments were amputation or local wide resection and sufficient radiation therapy, which often caused the lifelong disability of patients, the survival rate of less than $20 \%$, as well as very high recurrence rate. After adjuvant chemotherapy was added in the treatment, the prognosis of patients could be improved substantially.

Adjuvant chemotherapy normally means to use anti-tumor drugs after controlling the local tumor surgically for treating the micro lesions that may move to lungs, skeleton, lymph and other positions. As proved in extensive clinical practices, adjuvant chemotherapy is very effective for osteosarcoma 
and Ewing's sarcoma, and improves the 5-year survival rate significantly [1]. In the 1970s, the appearance of pre-operative chemotherapy could be regarded as another significant progress of chemotherapy, and it was later named neo-adjuvant chemotherapy. After that, chemotherapy could be also used to improve the limb salvage rate besides improving the survival rate of patients and lowering the local recurrence rate and metastasis rate. Rosen et al. [2-3] pointed out that neo-adjuvant chemotherapy is not a simple model of "preoperative chemotherapy + surgery + postoperative chemotherapy", but includes the overall assessment of patients and tumors after preoperative chemotherapy, which means to pay attention to whether the pain is alleviated, how much the mass shrinks, whether the lesion margin becomes clear in the medical imaging, whether osteoscherosis is worsened, and whether the new vessels of tumor decrease. After preoperative chemotherapy, the sample resected in the surgery is used for pathological classification. When patients have the tumor cell necrosis rate higher than $90 \%$ after chemotherapy, their 5-year survival rate will be up to $80 \%-90 \%$. When patients have the tumor cell necrosis rate lower than $90 \%$, their survival rate will be lower than $60 \%$ [4-7]. Hence, the postoperative chemotherapy scheme should be adjusted if the second condition occurs.

\section{Surgical Staging (Enneking Staging) of Musculoskeletal Tumor}

To select the surgery under the same medical parameters and compare their results, a surgical staging system should be used to improve the assessment of risk level and facilitate the information exchange and collaboration. A system was officially put forth by Enneking [7] in 1986, i.e. grade (G), tumor (T), and metastasis (M). Grade can be divided into low grade (G1) and high grade (G2), while tumors are classified into intra-compartmental (T1) and extra-compartmental (T2). The intra-compartmental tumors exist inside osseous tissues.

The staging system includes grade $(\mathrm{G})$, site $(\mathrm{T})$ and presence of local or distant metastases (M). The grade reflects the biological behavior and the degree of invasiveness, and indicates the risk of increasing extracapsular extension, the formation of satellite lesions, and the presence of regional and distant metastases, which can reveal the local recurrence and metastasis after surgery. The grade is determined on the basis of histological pattern, medical imaging representation, clinical course, biochemical test and quantitative histological examination. By using these data, pathological changes can be classified not G0-benign, G1-low grade malignant, and G2-high grade malignant. In terms of histology and radiology, benign pathological changes are well differentiated, free of transformed cells or nucleus division phase, intracapsular, without peripheral reaction area, subject to blunt pressure during growth, and rarely damaging natural barrier. Some highly invasive pathological changes may penetrate the capsule and invade the extracapsular tissues, but there are not satellite lesions, regional skip metastases or distant hematogenous or lymphatic metastases.

Staging aims to reveal the relative risk of different surgeries for a pathological change, and is not limited to offering a specific scheme for a type of tumor a patient has. Hence, it should take into account the age, gender, expectation and lifestyle of the patient, the purpose of the surgery, the skills and experience of the surgeon, and what adjuvant therapy is available to the patient, etc. The recurrence rate depends on the margin of surgery instead of local resection or amputation. At some dissection positions, it is easier to obtain the required margin. For instance, the recurrence rate after surgery at the upper thigh is twice higher than that at the lower thigh, while the recurrence rate on the palm side of forearm is twice greater than that at the back side of forearm.

\section{Limb Salvage Treatment}

The development of limb salvage treatment has been greatly promoted by the development of effective chemotherapy as well as the improvement of imaging technology and reconstruction technology. Limb salvage treatment follows the principle that local recurrence rate should not increase significantly compared with amputation. Whether tumor can be entirely resected with extensive surgical margin is crucial to local recurrence control, so surgical staging and determination 
of surgical margin are of great significant to preoperative plan and during surgery. Multi-center research has proved that limb salvage treatment causes only a small increase of local recurrence rate, and does not affect the overall survival rate of patients. The difference between survival rate of patients receiving limb salvage treatment and radical surgery is not statistically meaningful.

\subsection{Resection Principle}

As chemotherapy can narrow the surgical margin of tumors, wide resection can be implemented on this basis, i.e. optimal margin resection, which can not only keep the extremities that still function, but also achieves the radical cure for local tumor. The basic requirement of limb salvage treatment is the en bloc resection of tumor. In principle, a layer of normal tissue is kept in all directions of tumor, so this resection is generally regarded as wide resection, but it may be also performed in the form of marginal resection in some cases, especially between tumor and neuron or vessel. To prevent any leftover of tumor tissue and intra-operative spread, the resected tissues should, in principle, include tumor and its peripheral normal soft tissues as well as soft tissues around incision for biopsy. In other words, surgery is performed inside normal tissues to keep surgical devices from direct contract with tumor. Bone resection level should be $3-5 \mathrm{~cm}$ away from both ends of osteosarcoma, and the level can be determined on the basis of X-ray film, CT and MRI scanning.

Along with the progress of surgical technology, reconstruction materials and adjuvant treatments, some patients may receive the limb salvage treatment, which not only reconstructs the limb surgically, but also resects enough margin. During the process of reconstruction, it is often necessary to prepare large bone graft, internally-fixed prosthesis, or composite biological or artificial material. However, there is not perfect way of reconstruction available yet, and every existing treatment has its advantages and disadvantages. At present, osteoarticular allografting or reconstruction after inactivated bone replantation is often practiced. The treatment belongs to biological reconstruction, but allografting is subject to very high occurrence rate of postoperative complications, and inactivated tumor bone replantation causes very high local recurrence rate. Thanks to the improved curative rate of osteosarcoma, most patients can live for another 5 years and even a longer time, which requires a way of reconstruction for long-term use. Successful arthrodesis can realize the joint fusion with good stability and durability after joint resection, but it takes a longer time to heal, and is often unable to satisfy patients' needs for functions.

\subsection{Bone Allografting}

Large bone allograft has been widely applied in large reconstruction treatment. Large bone bank offers the bone allograft of different models and sizes. These bone grafts allow the attachment of soft tissues to retain the function of muscles and maintain the stability of joints. Bone grafts can provide the regeneration skeleton for living tissues. What is more important, they can make all normal connecting tissues (including living bones, cartilages, tendons and ligaments) full of matrixes to maintain the integrated structure of tissues. The main problems of bone allograft include fracture, nonunion, and infection [8]. The overall occurrence rate of fracture is around $16 \%-19 \%$, while more than $75 \%$ of patients can be treated with internal fixation or bone grafting after fracture. The bone graft is incorporated to host bone more slowly than the incorporation between normal bones. The occurrence rate of bone graft nonunion is around $17 \%$, and it is impossible to identify normal union, delayed union and nonunion. Among them, half of the patients experience union after bone grafting or other treatment. The occurrence rate of infection is $6 \%-13 \%$. As graft contains no blood vessel, it is very difficult to radically eliminate the infection, so most patients have to take out their grafts. In this case, the infection of large pelvic grafts happens more often. The occurrence rate of infection can be reduced by placing the muscles and soft tissues with good blood supply around the graft, especially at any position with thin skin, e.g. proximal tibia. The acrylic cement soaked with antibiotics can be used to lower the occurrence rate of infection, and slow down the resorption of bone.

\subsection{Bone Autografting}

Autograft can prevent the occurrence of immune reaction and the spread of infectious diseases, but it is not used so extensively as allograft to treat large bone defects. Autogenous bones should be gathered by using a separate set of devices and gloves, and the bone gathering area should be not be 
contaminated by tumor. The commonest bone gathering area is the cancellous bone of ilium, which was often used to stimulate the formation of new bone and facilitate the healing of fracture in the past. The infection rate of autograft is lower than that of large non-vascularized graft. Vascularized autograft can be used in local grafting or free grafting. The commonest free grafting is free vasculafized fibular grafting. Due to the existence of blood supply, vascularized graft has some advantages theoretically, e.g. less possibility of infection, less fatigue damage, and possible repair of hyperplasia.

\subsection{Inactivated Replantation}

If a tumor does not cause severe bone damage, the bone section with tumor can be resected and inactivated, and then put back into the defective position. The implantation of inactivated bone is the main method for reconstruction in some countries that have no large bone bank and whose internally-fixed prosthesis is relatively expensive. Inactivated bone also has some value for use even in developed countries as it may be greatly suitable in terms of immunity and structure. To use such kind of bone, inactivation must be carried out strictly by exposing it to high temperature for a long time, freezing it in liquefied nitrogen repeatedly, and placed it under extensive radioactive rays. When inactivation kills all the tumor cells, it may lower the quality of graft to different degrees. For instance, freezing in liquefied nitrogen may reduce the mechanical strength of bone significantly, and inactivate bone morphogenetic proteins. At present, the commonest method for inactivation in Japan and South Korea, etc. is the so-called pasteurization process, which immerses bone in salt water of around $60^{\circ} \mathrm{C}$ for $30 \mathrm{~min}$. It is reported that this process can retain the bone morphogenetic protein while inactivating the tumor bone.

\subsection{Artificial Prosthesis Replacement}

After tumor resection, the reconstruction of skeleton often requires large bone graft, internally-fixed prosthesis, or composite biological or artificial material. At present, the artificial prosthesis reconstruction method for large bone defects after malignant bone tumor has been widely accepted, and the reconstruction with metallic prosthesis has been practiced in more and more cases. Compared with other reconstruction methods, the reconstruction with metallic prosthesis has the following advantages: durable internal fixation, good stability immediately after surgery, good short-term and long-term functional prognosis, and good motion of joints after surgery. The most important advantage is the lower occurrence rate of postoperative complications than allograft bone reconstruction. The use of internally-fixed prosthesis in joints is advantageous as the stability of skeletal muscles and the motion of joints can be immediately restored without bone nonunion, and patients can move extremities without waiting for osteoporotic healing, which is very important to the patients with a short survival period. Like allograft, internally-fixed prosthesis replacement is accompanied by lots of complications [9-II]. The occurrence rate of infection is $2 \%-9 \%$, and it is often necessary to take out the prosthesis. The occurrence rate of infection varies in different dissection areas, and is the highest at proximal tibia (due to the fewest soft tissues). The muscle flap of gastrocnemius should be used as much as possible to transplant the prosthesis covering tibia, so as to reduce the occurrence rate of infection. Prosthesis replantation often leads to deep infection, so it is necessary to perform surgical debridement, place the acrylic cement temporarily soaked with antibiotics, and use venous antibiotics, etc. The failure of prosthesis is mainly caused by aseptic loosening, which is a specific issue of internally-fixed prosthesis [12-13] and a major complication of prosthesis reconstruction. As reported in references, the occurrence rate of aseptic loosening is $0-56 \%$, and related to the position of prosthesis. On the whole, the prosthesis of proximal tibia has the highest failure rate, while the prosthesis of proximal femur has the best prognosis [14-15]. The design and fixation of prosthesis can be improved to reduce the rate of loosening. For instance, the occurrence rate of aseptic loosening can be effectively reduced by selecting cement stem, and designing hydroxyapatite (HA) coating at the collar of prosthesis connected to bone. Bone grafting at the connection between host bone and prosthesis can promote the formation of extracortical bone bridge, which can prevent the fragments of prosthesis from entering the bone lining interface, so as to prevent aseptic loosening. Additionally, children suffer from aseptic loosening more often than grownups. At 
present, some scholars believe that aseptic loosening is the process causing the complications of prosthesis instead of its result, and lack of active treatment for aseptic loosening will inevitably result in lining wear, broken prosthesis and other mechanical complications. A prosthesis featured by reduced stress can have an improved service life. Conventional prosthesis allows the motion of joint facing a direction only, so its stem is under heavy pressure, and often loosened. The new-style prosthesis with rotating hinge can move in all directions, so it is subject to uniform stress.

\section{Existing Problems}

Misdiagnosis and mistherapy is a very serious problem for two reasons: 1 . the incidence of bone and soft tissue tumors is lower, so some surgeons do not pay much attention to these tumors, and lack the experience in their clinical diagnosis and therapy; 2. clinical practice does not follow the existing standards for diagnosis and therapy. Misdiagnosis and mistherapy may cause the following hazards: the curettage inside lesions is performed by regarding malignant tumor as benign tumor, which causes local contamination and spread of tumor. This makes it very difficult to achieve local wide resection in another surgery, and significantly lowers the possibility of cure for patients, so the patients may have to suffer from cruel amputation.

Wide resection of malignant bone tumor has two meanings, i.e. understanding about the concept of wide resection, and specific operation of wide resection. As different surgeons may have different operational skills, wide resection will lead to different results. According to the requirements of the International Society of Limb Salvage (ISOLS), the local recurrence rate of limb salvage treatment should be controlled to be lower than $8 \%$, so limb salvage treatment should be practiced by surgeons skilled in wide resection. Moreover, the scope of resection in the surgery may be insufficient as the importance of safe margin concept, surgical staging and surgical margin is not sufficiently addressed in the preoperational plan.

The existing problems of chemotherapy include nonstandard preoperative chemotherapy and postoperative chemotherapy. The most significant role of preoperative chemotherapy is to provide the in vivo test for the effectiveness of chemotherapeutic drugs to tumors. After surgical resection, the necrosis rate of tumor is examined. Any patients with higher necrosis rate will enjoy 5-year survival rate. If patients have poor reaction during chemotherapy, it is recommended to change the chemotherapeutic drugs. Nonstandard medication is mainly reflected in insufficient dosing. Most doctors responsible for chemotherapy are not specialized in bone tumors, so they are not familiar with the toxicity and pharmacological process of drug, and use insufficient dosing for safety. For this reason, chemotherapy cannot achieve the desired effect, lowering the survival rate of patients.

The methods for joint reconstruction after tumor resection have their advantages and disadvantages. The reconstruction with bone and joint graft belongs to biological reconstruction, but bone allografting often results in postoperative complications. Additionally, most patients with malignant bone tumors need effective postoperative chemotherapy, so the functions should be restored as early as possible while implementing the reconstruction treatment. At present, the reconstruction with metallic artificial prosthesis for large bone defects after malignant bone tumor resection has been a widely recognized method. Most Chinese doctors of orthopedics are not very familiar with the operative skills and joint characteristics in the tumor-induced artificial joint replacement, including replacement position, way of prosthesis fixation, postoperative complications and prosthesis survival period, which causes such problems as high occurrence rate of operative complications and short prosthesis survival period. Therefore, it is very essential to encourage more Chinese doctors of orthopedics to learn the operative skills and relevant professional knowledge about tumor-induced artificial joint replacement.

\section{Acknowledgments}

This work was supported by The study of c-Myc and mTOR inhibitors in the combined targeting therapy of osteosarcoma. Jilin provincial finance department (No 3D517P373430). 


\section{References}

[1]. Rajiah P, Ilaslan H, Sundaram M. Imaging of primary malignantbone tumors (nonhematological). Radiol Clin North Am, 2011, 49(6):1135-1161.

[2]. [2]Boulet B, Caramella C, Couanet D, et al. MR imaging patterns of bonemarrow. J Radiol, 2010, 91(9): 935-949.

[3]. Hwang S. Imaging of lymphoma of the musculoskeletal system. MagnReson Imaging Clin N Am, 2010, 18(1): 75-93.

[4]. Daniel A Jr, Ullah E, Wahab S, et al. Relevance of MRI in predictionof malignancy of musculoskeletal system: a prospective evaluation.BMC Musculoskelet Disord, 2009, 10(10): 125.

[5]. Ginat DT, Mangla R, Yeaney G, et al. Diffusion-weighted imagingfor differentiating benign from malignant skull lesions and correlationwith cell density. AJR Am J Roentgenol, 2012, 198(6): 597-601.

[6]. Costa FM, Canella C, Gasparetto E. Advanced magnetic resonanceimaging techniques in the evaluation of musculoskeletal tumors.Radiol Clin North Am, 2011,49(6): 1325-1358.

[7]. $\mathrm{Xu} \mathrm{WJ}$, Chen HS. Attention shall be paid to the study and applicationof MR imaging on musculoskeletal system. Chin J Magn ResonImaging, 2012, 3(4): 241-244.

[8]. Chen YM, Meng QF, Jiang B, et al. Periosteal anomaly inosteosarcoma: the imaging fi ndings and its pathological basis. Chin JRadiol, 2008, 42(3): 247-252.

[9]. Bloem JL, Reidsma II. Bone and soft tissue tumors of hip and pelvis.Eur J Radiol, 2012, 81 (12): 3793-3801.

[10]. Fayad LM, Mugera C, Soldatos T, et al. Technical innovationin dynamic contrast enhanced magnetic resonance imaging ofmusculoskeletal tumors: an MR angiographic sequence using a sparsek-space sampling strategy. Skeletal Radiol, 2013, 42(7): 993-1000.

[11]. f'Tink SJ, Rutledge J, Lewis VO, et al. favorable long\} erm results of prosthetic aTthroplasty of the knee for distal femur neoplasms)].Clin Or- thop Relat Res, 2005, 438:65-70.

[12]. Gnwin PS, Cannon SR, Grimer RJ, et al. Aseptic loosening in cemented custom $\}$ nade prosthetic replacement for bone tumours of the lower limb ].J Bone Joint Surg, 1996, 78 (1):5-13.

[13]. Zeegen L,N, Aponte=finao LA, llornicek fJ, et al. Survivorship analysis of 141 modular metallic endoprostheses at early followup)].Clin Or-thop Relat Res, 2004, 420:239-250.

[14]. KabukCITOglu Y, GT'1meT' \}, \}1\}11lnlan RM, et al. LndOpT'OSthetlC T'eplaCement for' pT7n1aT'y nlahgnant tum OT'S Of the pT'Oxlnlal femur'].Clin Orthop Relat Res\} 1999 358:8-14.

[15]. Bickels J, Wittig JC, Kollender Y, et al. Distal femur resection with endoprosthetic reconstruction].Clin Orhop Relat Res, 2002, 400: 225-235. 